\title{
Pulmonary embolism and screening for concomitant proximal deep vein thrombosis in noncritically ill hospitalized patients with coronavirus disease 2019
}

\author{
Álvaro Dubois-Silva ${ }^{1,2}$ (Cristina Barbagelata-López ${ }^{1}$ Álvaro Mena ${ }^{2,3} \cdot$ Patricia Piñeiro-Parga ${ }^{1} \cdot$ \\ Diego Llinares-García ${ }^{1} \cdot$ Santiago Freire-Castro ${ }^{1}$
}

Received: 12 May 2020 / Accepted: 20 June 2020 / Published online: 26 June 2020

(c) Società Italiana di Medicina Interna (SIMI) 2020

Keywords Coronavirus disease $2019 \cdot$ Pulmonary embolism · Deep vein thrombosis $\cdot$ Screening $\cdot$ Coagulopathy

Dear Editor,

Coagulation abnormalities with elevated D-dimer levels have been identified as predictors of poor prognosis in patients with coronavirus disease 2019 (COVID-19) in China [1-4]. The correlation between D-dimer levels and diagnosis and prognosis of venous thromboembolism (VTE) has been widely described $[5,6]$. Thus, many questions regarding the relationship between these entities are emerging [7].

Recently, a VTE prevalence of $25 \%$ has been reported in patients with severe novel coronavirus pneumonia [8]. However, there is limited information about the clinical characteristics of noncritically ill hospitalized patients with COVID-19 who develop pulmonary embolism (PE), or their prevalence of lower limb concomitant deep-vein thrombosis (DVT). Therefore, the purpose of this study was to analyze the clinical data and assess the prevalence of concomitant proximal DVT in COVID-19 nonintensive care unit (nonICU) patients with PE.

Álvaro Dubois-Silva

alvarodibua@gmail.com

1 Department of Internal Medicine, Complexo Hospitalario Universitario de A Coruña (CHUAC), Servizo Galego de Saúde (SERGAS), A Coruña, Spain

2 Universidade da Coruña (UDC), A Coruña, Spain

3 Infectious Diseases Unit, Department of Internal Medicine, Complexo Hospitalario Universitario de A Coruña (CHUAC), Servizo Galego de Saúde (SERGAS), A Coruña, Spain

\section{Methods}

This cross-sectional observational study describes non-ICU patients hospitalized for COVID-19 in a single center in A Coruña (northwest Spain) during April 2020 who developed a PE. Patients met inclusion criteria if they were $\geq 18$ years old, had a confirmed diagnosis of severe acute respiratory syndrome coronavirus-2 (SARS-CoV-2) infection and were diagnosed with PE. Exclusion criteria were ICU admission at enrollment (i.e., a previous ICU stay was allowed), history of VTE within the past 3 months, and presence of a venous malformation or anatomical abnormality which hindered a reliable point-of-care compression ultrasonography (CUS) of the lower limbs. All patients (or their health-care proxies) provided informed consent for their study participation. The research protocol was conducted in accordance with the 2013 Declaration of Helsinki and the local Ethics Committee requirements.

COVID-19 was diagnosed according to World Health Organization interim guidelines [9] and confirmed by RNA detection of the SARS-CoV-2 and/or serological testing. PE diagnosis was established by positive contrast-enhanced, PE-protocol, helical chest computerized tomography (CT).

Point-of-care CUS of the lower limbs was performed to assess the prevalence of concomitant proximal DVT. Prior to this, risk factors for VTE and symptoms of PE and DVT were assessed using a simple questionnaire. Trained clinicians who were caring for these COVID-19 patients conducted lower limb CUS in safe conditions (i.e., with personal protective equipment). Proximal DVT diagnosis was established if a noncompressible vein segment was detected at or above the popliteal vein on CUS. Distal DVT (i.e., below the knee) was not screened using this protocol, according to our purpose of exclude only thrombosis of the larger (i.e. proximal) veins. 
Clinical data (demographics, comorbidities, concurrent medications, laboratory, and imaging tests) were recorded at baseline and during hospitalization until PE diagnosis. VTE was considered secondary to immobilization if it appeared within 2 months of confinement to bed with bathroom privileges for $\geq 4$ days. Immobilization due to COVID-19 prior to VTE was included in this definition. D-dimer was measured by automated latex-enhanced HemosIL D-Dimer (Instrumentation Laboratory, Bedford, MA, USA) immunoassay.

Patients were managed according to the hospital's clinical practice, with no standardizations or treatment recommendations. Diagnostic tests for PE were only applied when PE was clinically suspected. Lower-limb CUS examination was performed in all patients regardless of the presence or absence of DVT symptoms. All data were recorded electronically and collected retrospectively using standardized case report forms. Continuous variables are expressed using the median and interquartile ranges (IQRs). SPSS software (v. 22.0; IBM SPSS, Armonk, NY, USA) was used for all analyses.

\section{Results}

From April 2 to April 17, 2020, a total of 171 patients remained hospitalized with confirmed COVID-19 in the Internal Medicine Department of the University Hospital of A Coruña. Among these, 8 (4.68\%; 95\% CI 2.39-8.96) patients diagnosed with PE participated in the study. The patient characteristics are presented in Table 1. Seven (87.5\%) participants had pneumonia with bilateral pulmonary infiltration on imaging tests performed before PE diagnosis (Table 1). The remaining patient was diagnosed with bilateral pneumonia in the same chest CT scan which demonstrated the PE.

All patients were immobilized due to COVID-19 as the main risk factor for VTE. They received thromboprophylaxis with enoxaparin or biosimilar at a median daily dose of $40 \mathrm{mg}$ from admission to PE diagnosis (Table 2). The prophylactic dose was increased in $3(37.5 \%)$ patients due to rising D-dimer levels before (i.e. more than $24 \mathrm{~h}$ ) PE diagnosis, from 40 to $60 \mathrm{mg} /$ day in two patients and from 40 to $100 \mathrm{mg} /$ day in one (bellow their respective full therapeutic dose regimen). Contrast-enhanced helical chest CT was ordered because D-dimer levels were increasing (this was the only reason for one patient), while PE symptoms and/or respiratory worsening were also considered in the decisions for the remaining patients.

Six patients (75\%) had a Pulmonary Embolism Severity Index (PESI) at or above class III (Table 2). None of these patients had imaging findings suggesting right-ventricle (RV) dysfunction by CT-scan, neither by echocardiography or by laboratory findings among tested participants.
Table 1 Clinical characteristics of non-intensive care unit hospitalized patients with coronavirus disease 2019 and pulmonary embolism

COVID-19 and PE
Patients, $N$

Clinical characteristics,

Men

Age (median years, IQR)

Body weight (median kg, IQR)

Body mass index (median $\mathrm{kg} / \mathrm{m}^{2}$, IQR)

Charlson comorbidity index $\leq 1$

Concomitant diseases,

Hypertension

Dyslipidemia

Diabetes

Obesity

Current smoking

Chronic lung disease

Chronic heart failure

Chronic kidney disease

Recent major bleeding

Thombophilia

Concomitant therapies at admission,

Anticoagulants

Antiplatelets

Corticosteroids

NSAIDs

Immunosuppressive drugs

ACEI/ARB

COVID-19 characteristics,

Pneumonia

Bilateral pulmonary infiltration

Basal SpO2<93\%

ICU stay prior to PE diagnosis

COVID-19-related therapies,

Hydroxichloroquine

Antibiotics

Lopinavir/ritonavir

Corticosteroids

Tocilizumab

Remdesivir

Interferon
8

$5(62.5 \%)$

$67(58-74)$

75 (67-83)

$24.6(23.5-27.2)$

$7(87.5 \%)$

$3(37.5 \%)$

$4(50 \%)$

$1(12.5 \%)$

$1(12.5 \%)$

0

0

0

0

0

0

0

0

0

0

0

$3(37.5 \%)$

$7(87.5 \%)$

$7(87.5 \%)$

$5(62.5 \%)$

$2(25 \%)$

$7(87.5 \%)$

$8(100 \%)$

$7(87.5 \%)$

$7(87.5 \%)$

$5(62.5 \%)$

0

$2(25 \%)$
COVID-19 coronavirus disease 2019, PE pulmonary embolism, IQR interquartile range, NSAIDs non-steroidal anti-inflammatory drugs, $A C E I$ angiotensin-converting enzyme inhibitors, $A R B$ angiotensin II receptor blockers, $\mathrm{SpO} 2$ oxygen saturation as measured by pulse oximetry, ICU intensive care unit

All patients received full-dose of enoxaparin or biosimilar when PE was diagnosed, and none required thrombolysis. Only one patient had $\geq 4$ points in the sepsis-induced coagulophaty (SIC) score proposed by the International Society of Thrombosis and Haemostasis (ISTH). 
Table 2 Venous

thromboembolism-related characteristics of non-intensive care unit hospitalized patients with coronavirus disease 2019 and pulmonary embolism

\begin{tabular}{|c|c|}
\hline & COVID-19 and PE \\
\hline Patients, $N$ & 8 \\
\hline \multicolumn{2}{|l|}{ Risk factors for VTE, } \\
\hline Active cancer & 0 \\
\hline Surgery & 0 \\
\hline Immobility for $\geq 4$ days & $8(100 \%)$ \\
\hline Use of estrogens & 0 \\
\hline Pregnancy/postpartum & 0 \\
\hline None of the above & 0 \\
\hline Leg varicosities & $3(37.5 \%)$ \\
\hline Prior VTE & 0 \\
\hline \multicolumn{2}{|l|}{ Thromboprophylaxis, } \\
\hline Enoxaparin or biosimilar & $8(100 \%)$ \\
\hline Median enoxaparin or biosimilar dose (mg/day, IQR) & $40(40-60)$ \\
\hline Dose increase during thromboprophylaxis & $3(37.5 \%)$ \\
\hline Other anticoagulants & 0 \\
\hline \multicolumn{2}{|l|}{ Clinical symptoms and signs at PE presentation, } \\
\hline Chest pain & $1(12.5 \%)$ \\
\hline Syncope & 0 \\
\hline Worsening dyspnea & $5(62.5 \%)$ \\
\hline Hemoptysis & $1(12.5 \%)$ \\
\hline None of the above & $3(37.5 \%)$ \\
\hline Heart rate $>110 / \min$ & 0 \\
\hline $\mathrm{SBP}<100 \mathrm{~mm} \mathrm{Hg}$ & 0 \\
\hline $\mathrm{PaO} 2 / \mathrm{FiO} 2$ (median mm Hg, IQR) & $233(169-323)$ \\
\hline RV dysfunction (by imaging and/or laboratory tests) & 0 \\
\hline PESI $\geq$ class III & $6(75 \%)$ \\
\hline sPESI $\geq 1$ point & $6(75 \%)$ \\
\hline SIC score $\geq 4$ points & $1(12.5 \%)$ \\
\hline \multicolumn{2}{|l|}{ PE involvement, } \\
\hline Subsegmental & $4(50 \%)$ \\
\hline Segmental & $5(62.5 \%)$ \\
\hline Lobar & $1(12.5 \%)$ \\
\hline Main & 0 \\
\hline Central & 0 \\
\hline \multicolumn{2}{|l|}{ Time from COVID-19 to PE diagnosis, days } \\
\hline Median days (IQR) from COVID-19 symptoms onset & $19(17-23)$ \\
\hline Median days (IQR) from admission & $13(8-15)$ \\
\hline \multicolumn{2}{|l|}{ Laboratory tests, } \\
\hline Anemia & $5(62.5 \%)$ \\
\hline White blood cell count $\geq 10,000 / \mathrm{mm}^{3}$ & $4(50 \%)$ \\
\hline Lymphocyte count $<1000 / \mathrm{mm}^{3}$ & $4(50 \%)$ \\
\hline Platelet count $<150,000 / \mathrm{mm}^{3}$ & $1(12.5 \%)$ \\
\hline $\mathrm{INR} \geq 1.2$ & $1(12.5 \%)$ \\
\hline $\mathrm{CrCl}<60 \mathrm{~mL} / \mathrm{min}$ & 0 \\
\hline C-reactive protein $\geq 1 \mathrm{mg} / \mathrm{dL}$ & $6(75 \%)$ \\
\hline Serum ferritin $\geq 300 \mathrm{mg} / \mathrm{dL}$ & $7(87.5 \%)$ \\
\hline Lactate dehydrogenase $\geq 250 \mathrm{U} / \mathrm{L}$ & $7(87.5 \%)$ \\
\hline IL- $6 \geq 5 \mathrm{pg} / \mathrm{mL}$ & $8(100 \%)$ \\
\hline D-dimer levels (median ng/mL, IQR) & $10,000(5979-34,974)$ \\
\hline D-dimer levels $\geq 2000 \mathrm{ng} / \mathrm{mL}$ & $8(100 \%)$ \\
\hline D-dimer levels $\geq 5000 \mathrm{ng} / \mathrm{mL}$ & $7(87.5 \%)$ \\
\hline
\end{tabular}


Table 2 (continued)

COVID-19 and PE

Concomitant DVT

Signs or symptoms of lower-limb DVT

0

Proximal DVT of the lower limbs on CUS

0

COVID-19 coronavirus disease 2019, PE pulmonary embolism, VTE venous thromboembolism, IQR interquartile range, $S B P$ systolic blood pressure, $\mathrm{PaO} 2$ arterial oxygen tension, $\mathrm{FiO} 2$ fraction of inspired oxygen, $R V$ right ventricle, $P E S I$ pulmonary embolism severity index, $s P E S I$ simplified pulmonary embolism severity index, SIC sepsis-induced coagulopathy, INR international normalized ratio, $\mathrm{CrCl}$ creatinine clearance, $I L-6$ interleukin-6, DVT deep-vein thrombosis, CUS compression ultrasonography

Seven $(87.5 \%)$ patients had only segmental or subsegmental PE events, while one patient also had unilateral lobar involvement (Table 2). Time from onset of coronavirus disease 2019 symptoms to pulmonary embolism diagnosis in each patient is depicted in Fig. 1. Laboratory tests at PE diagnosis are summarized in Table 2. One patient did not have serum ferritin determination. None of these patients had concomitant proximal DVT of the lower limbs on CUS and none had DVT symptoms.

\section{Discussion}

Previous studies have reported a prevalence of proximal DVT of the lower limbs around 50\% in patients with PE, among whom the presence of DVT symptoms ranges from 42 to $48 \%[10,11]$ However, these studies did not assess the correlation between the PE-involved territory and the occurrence of concomitant DVT. We did not detect any concomitant proximal DVT of the lower limbs in our small sample of noncritically ill patients with COVID-19 and PE. Interestingly, all our patients had segmental of subsegmental events. Only one patient had more proximal involvement represented by a single unilateral lobar clot. Furthermore, most patients showed high levels of D-dimer, serum ferritin, interleukin-6, C-reactive-protein and lactate dehydrogenase in the COVID-19 context. Thus, our findings suggest a predominance of small-vessel thrombosis secondary to inflammatory and immune responses in these patients. Lower-limb CUS in the absence of DVT symptoms could probably be avoided in this patient profile.

One study reported lower mortality in severe COVID-19 patients who were treated with heparin and had an ISTH SIC score $\geq 4$ or a D-dimer $>3000 \mathrm{ng} / \mathrm{mL}$ [12]. Only two of our patients had a previous ICU stay, and all patients received thromboprophylaxis with enoxaparin or biosimilar continuously from their first day of admission. D-dimer levels were $>5000 \mathrm{ng} / \mathrm{mL}$ in almost all (7) of these lesssevere COVID-19 patients at PE diagnosis. Nevertheless, only one patient met SIC criteria with thrombocytopenia. Hence, mechanisms other than classic SIC or disseminated intravascular coagulopathy could be involved in the smallvessel thrombosis of the COVID-19 patients with the profile we have described herein. Other authors have also noticed a different coagulopathy pattern to SIC, [13] while the role of endothelial dysfunction in the microvascular thrombosis that we detected was suggested by others [14]. These potential mechanisms must be addressed in further studies.

Although PE severity represented by the PESI and sPESI was high in our sample, we note that some features

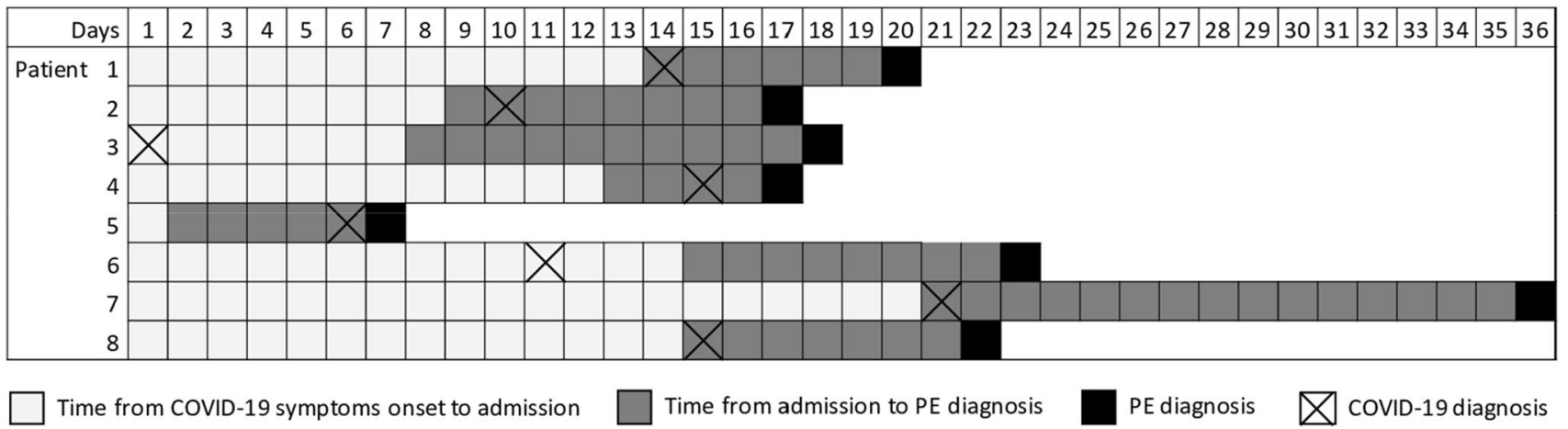

Fig. 1 Time from onset of coronavirus disease 2019 symptoms to pulmonary embolism diagnosis in each patient. COVID-19 coronavirus disease 2019, $P E$ pulmonary embolism 
of these indices (i.e., hypotension, pulse rate $\geq 110$ beats per minute, respiratory rate $\geq 30$ breaths per minute, altered mental status and $\mathrm{SpO} 2<90 \%$ ) would be present in many patients with SARS-CoV-2 pneumonia. In addition, we detected PE with low thrombotic burden, no RV dysfunction, and a poor evolution of coronavirus pneumonia in the same chest CT scan in most of our patients. Consequently, we hypothesize that many of these patients would have respiratory deterioration not explained only by the PE. PESI could be insufficiently accurate for assessing PE severity in patients with worsening pneumonia; thus, special attention to changes in their condition might be warranted when PE is suspected in COVID-19 patients.

Our study is not without limitations. First, we have described a relatively small series of patients with COVID-19 and PE at a single center in Spain. Therefore, our findings must be interpreted cautiously and require external validation in larger cohorts. Second, since this was an observational study without prospective followup from PE diagnosis, randomized control trials will be needed before recommendations can be made on the clinical management of these patients. Third, although no signs of RV dysfunction were detected by CT-scan, echocardiography or laboratory tests to assess RV function were not performed in all patients. Hence, data regarding RV dysfunction could be insufficiently accurate.

In summary, the study results show that a subset of non-ICU hospitalized COVID-19 patients diagnosed with PE has chiefly segmental or subsegmental events without concomitant proximal DVT of the lower limbs. Our findings suggest a predominance of small-vessel thrombosis secondary to inflammatory and immune responses in these patients.

Acknowledgements We appreciate the support of all staff who is working in Complexo Hospitalario Universitario de A Coruña (A Coruña, Spain).

Author contributions AD-S contributed to the concept and design of the study, compression ultrasound examination, analysis and acquisition of data, interpretation of the results and drafting of the manuscript. CB-L contributed to the concept and design of the study, compression ultrasound examination and reviewing of the manuscript. AM contributed to the concept and design of the study, statistical analysis and drafting of the manuscript. PP-P contributed to the compression ultrasound examination, acquisition of data and reviewing of the manuscript. DL-G and SF-C contributed to the compression ultrasound examination and reviewing of the manuscript. All authors approved the final version of the manuscript.

Funding The present work was carried out at the Department of Internal Medicine of the Complexo Hospitalario Universitario de A Coruña (A Coruña, Spain), and was supported in part by grants from Fondo de Investigación Sanitaria (JR17/00028).

\section{Compliance with ethical standards}

Conflict of interest The authors declare that they have no conflict of interest.

Statement of human and animal rights and ethics approval This study was conducted in accordance with the 1964 Helsinki Declaration and its later amendments and the local Ethics Committee requirements. Ethical approval was waived in view of the retrospective nature of the study and all the procedures being performed were part of the routine care.

Informed consent Informed consent was obtained from all individual participants included in the study.

\section{References}

1. Zhou F, Yu T, Du R et al (2020) Clinical course and risk factors for mortality of adult inpatients with COVID-19 in Wuhan, China: a retrospective cohort study. Lancet 395:1054-1062. https://doi.org/10.1016/S0140-6736(20)30566-3

2. Tang N, Li D, Wang X, Sun Z (2020) Abnormal coagulation parameters are associated with poor prognosis in patients with novel coronavirus pneumonia. J Thromb Haemost. https://doi. org/10.1111/jth.14768

3. Guan W-J, Ni Z-Y, Hu Y et al (2020) Clinical characteristics of coronavirus disease 2019 in China. N Engl J Med. https://doi. org/10.1056/NEJMoa2002032

4. Lippi G, Favaloro EJ (2020) D-dimer is associated with severity of coronavirus disease 2019: a pooled analysis. Thromb Haemost. https://doi.org/10.1055/s-0040-1709650

5. Kelly J, Rudd A, Lewis RR, Hunt BJ (2002) Plasma D-dimers in the diagnosis of venous thromboembolism. Arch Intern Med 162:747-756. https://doi.org/10.1001/archinte.162.7.747

6. Palareti G, Cosmi B, Legnani C et al (2006) D-dimer testing to determine the duration of anticoagulation therapy. N Engl J Med 355:1780-1789. https://doi.org/10.1056/NEJMoa054444

7. Wang T, Chen R, Liu C et al (2020) Attention should be paid to venous thromboembolism prophylaxis in the management of COVID-19. Lancet Haematol 2019:2019-2020. https://doi. org/10.1016/S2352-3026(20)30109-5

8. Cui S, Chen S, Li X et al (2020) Prevalence of venous thromboembolism in patients with severe novel coronavirus pneumonia. J Thromb Haemost. https://doi.org/10.1111/jth.14830

9. World Health Organization Clinical management of severe acute respiratory infection (SARI) when COVID-19 disease is suspected: Interim guidance V 1.2. In: Publ. March 13, 2020. https://www.who.int/publications-detail/clinical-management -of-severe-acute-respiratory-infection-when-novel-coronaviru s-(ncov)-infection-is-suspected. Accessed 14 Apr 2020

10. Jiménez D, Aujesky D, Díaz G et al (2010) Prognostic significance of deep vein thrombosis in patients presenting with acute symptomatic pulmonary embolism. Am J Respir Crit Care Med 181:983-991. https://doi.org/10.1164/rccm.200908-1204OC

11. Hirmerova J, Seidlerova J, Chudacek Z (2018) The prevalence of concomitant deep vein thrombosis, symptomatic or asymptomatic, proximal or distal, in patients with symptomatic pulmonary embolism. Clin Appl Thromb 24:1352-1357. https:// doi.org/10.1177/1076029618779143 
12. Tang N, Bai H, Chen X et al (2020) Anticoagulant treatment is associated with decreased mortality in severe coronavirus disease 2019 patients with coagulopathy. J Thromb Haemost. https://doi.org/10.1111/jth.14817

13. Levi M, Thachil J, Iba T, Levy JH (2020) Coagulation abnormalities and thrombosis in patients with COVID-19. Lancet Haematol 7:e438-e440. https://doi.org/10.1016/S2352 $-3026(20) 30145-9$

14. Sardu C, Gambardella J, Morelli MB et al (2020) Hypertension, thrombosis, kidney failure, and diabetes: is COVID-19 an endothelial disease? a comprehensive evaluation of clinical and basic evidence. J Clin Med. https://doi.org/10.3390/jem9051417

Publisher's Note Springer Nature remains neutral with regard to jurisdictional claims in published maps and institutional affiliations. 